\title{
Hibiscus Tea and Health: A Scoping Review of Scientific Evidence
}

\author{
Christopher J Etheridge ${ }^{1}$, and Emma J Derbyshire ${ }^{2 *}$ \\ ${ }^{1}$ Integrated Herbal Healthcare, London, United Kingdom \\ ${ }^{2}$ Nutritional Insight, Epsom, Surrey, United Kingdom
}

*Corresponding author: Emma J Derbyshire, Nutritional Insight, Epsom, Surrey, United Kingdom, E-mail: emma@nutritional-insight.co.uk

Received: 18 Jun, 2020 | Accepted: 10 Jul, 2020 | Published: 27 Jul, 2020

Citation: Etheridge CJ, Derbyshire EJ (2020) Hibiscus Tea and Health: A Scoping Review of Scientific Evidence. Nutr Food Technol Open Access 6(2): dx.doi.org/10.16966/2470-6086.167

Copyright: (c) 2020 Etheridge CJ, et al. This is an open-access article distributed under the terms of the Creative Commons Attribution License, which permits unrestricted use, distribution, and reproduction in any medium, provided the original author and source are credited.

\begin{abstract}
Over the last few decades, health evidence has been building for hibiscus tea (Hibiscus sabdariffa L. Malvaceae). Previous reviews show promise in relation to reducing cardiovascular risk factors, hypertension and hyperlipidaemia, but broader health perspectives have not been widely considered. Therefore, a scoping review was undertaken to examine the overall health effects of hibiscus tea. A PubMed search was undertaken for metaanalysis (MA) and systematic review papers, human randomised controlled trials (RCT) and laboratory publications investigating inter-relationships between hibiscus tea and health. Twenty-two publications were identified (four systematic/MA papers, nine human RCT controlled trials and nine laboratory publications).Strongest evidence exists in relation to cardiovascular disease, suggesting that drinking 2-3 cups daily (each $\approx 240-250 \mathrm{~mL}$ ) may improve blood pressure and potentially serve as a preventative or adjunctive therapy against such conditions. Emerging evidence exists for favourable effects on lipid profiles, insulin resistance, oxidative stress and inflammation. Further research using larger and longer human studies is warranted.
\end{abstract}

Keywords: Hibiscus tea; Health; Antioxidants; Polyphenolic compounds; Cardiovascular disease; Blood Pressure

\section{Introduction}

Hibiscus tea is usually derived from the dried calyces of the tropical plant Hibiscus sabdariffa L. (HSL) which belongs to the mallow family (Malvaceae) [1]. It is thought to be a native of West Africa, but is commonly cultivated across the tropics and subtropics including in China, Egypt, Mexico, Thailand and the West Indies [2]. HSL is sometimes referred to in English as 'roselle' or 'red sorrel' and in Arabic as 'karkade' [3]. This naming is attributed to its colouring with the roselle calyx being a bright red colour and abundant in anthocyanins [4]. It is the calyces (outer parts of the flower) that are most commonly used worldwide, to make hot and cold infusions [3]. HSL is also rich in organic acids and thus is also referred to as 'sour tea' due to these providing a distinctive tart taste [5].

Hibiscus tea is becoming increasingly popular which largely appears to be attributed to its bioactive properties, which could benefit public health [6,7]. From a historical perspective HSL has been used in folklore and traditional medicines [8,9]. Its rich phytonutrient profile may be responsible for such reported health effects [1]. For example, the phenolic acid protocatechuic acid, organic acids (hydroxycitric acid and hibiscus acid) and anthocyanins (delphinidin-3-sambubioside and cyanidin-3-sambubioside) have been listed as important bioactive components within HSL [10]. A compositional analysis of Hibiscus calyces found these to be rich in lutein, chlorogenic acids and delphinidin 3-sambubioside [11]. HSL extracts have additionally been found to have antioxidant and antimicrobial properties [7].
A growing body of evidence suggests that herbal teas, including HSL could benefit aspects of preventative and clinical health [8]. For example, one MA [5] of five RCT comprised of 390 participants found that HSL had significant effects lowering both systolic and diastolic blood pressure. HSL extracts have also been found to have anti-diabetic, anti-hypertensive, nephro- and hepato-protective effects as well as potential benefits on lipid metabolism and renaldiuretic effects [10]. These actions have been ascribed to HSL extracts having a notable antioxidant profile, vasorelaxant effects and ability to inhibit $\alpha$-glucosidase, $\alpha$-amylase, angiotensin-converting enzymes and modulate calcium channels [10]. Other work has reported that HSL could also have potential anti-obesity actions [12,13]. A systematic review of ten studies concluded that HSL may have a role in the treatment of mild to moderate hypertension, although ongoing research is warranted [14].

Taking the growing evidence-base on board, the present scoping review aims to collate evidence from systematic reviews, MA papers, human trials and laboratory studies, investigating inter-relationships between hibiscus tea consumption and health.

\section{Methods and Materials}

\section{Study design}

Scoping reviews are useful for collating evidence on a topic-they are a form of knowledge synthesis and useful for answering broader questions, including what is known about a concept [15]. 
For the screening of human trials the Preferred Reporting Items for Systematic Reviews and Meta-Analyses (PRISMA) approach was used (Figure 1) [16]. Alongside this, the recently published PRISMA Extension for Scoping Reviews (ScR) checklist developed by a 26-member expert panel was also applied. [16] The quality of human trials was further determined using the validated Jadad criterion [17].

\section{Inclusion/Exclusion criteria}

The present scoping review analysed associations between hibiscus tea and markers of health. Included papers were published in Englishlanguage in peer-reviewed journals. Human trials were screened and used hibiscus delivered as teas, infusions or aqueous extracts (specifying the amount consumed) rather than concentrated extracts or supplements. Trials were limited to RCTs using the PubMed search restriction tab. RCTs are regarded as the 'gold-standard' for identifying causal relationships as they largely eliminate bias typically associated with other study designs [18].

Multi-interventions using combinations of tea infusions, medications, tea supplements, condensed extracts or other interventions that could potentially confound results were excluded when screening human studies. Interventions, however, where different tea forms were randomly allocated to separate groups of participants were included. No time restrictions were applied for the systematic, MA review or RCT search. A restriction of ten years was applied to the laboratory studies.

\section{Search strategy \& data charting}

The search for relevant studies was conducted using the PubMed database. For the identification of publications, the search terms "Hibiscus tea" or "Hibiscus sabdariffa tea" or "roselle tea" or "sour tea" were used and acknowledged as text words [tw] within the PubMed search strategy. Further to this, a manual search for publications was undertaken by screening the references listed in key studies. The search focused on gathering evidence from: (1) Prior systematic review and MA papers, (2) Human trials and (3) Laboratory publications (studies and reviews). Search restrictions were customised and limited to: Systematic reviews, MA papers, human RCTs and laboratory publications.

Studies were screened using the listed inclusion/exclusion criteria and identified by the two contributing authors. Studies were initially screened using their title. They were then further verified using the abstract. The procedure of identification, screening, evaluation of eligibility and inclusion is illustrated in Figure 1.

Data charted from the human trials included the following: General details of the study, participants, study design, tea intervention (type and dosage), health outcome(s) and main study findings with any reported significant p-values. For laboratory publications data relating to the study and its design (laboratory model) were extrapolated, Hibiscus intervention/focus and health outcomes were extracted along with the main findings.

\section{Results}

The search for articles identified 31 publications initially (seven MA papers, eight RCTs from PubMed, three RCTs from literature sources and 13 laboratory papers). Of these, three systematic/MA papers and four laboratory publications were irrelevant and two trials used supplements or medications. Subsequently 22 papers were eligible for inclusion within the review-four systematic/MA papers, nine human RCTs and nine laboratory studies (Figure 1).
Most of the articles focused on aspects of cardiovascular wellbeing (Table 1). Three concentrated on hypertension or blood pressure $[5,19,20]$ and two serum lipid levels $[21,22]$. Two MA studies found that both sour tea and HSL significantly reduced systolic and diastolic blood pressure $[5,19]$. Significant reductions in plasma glucose levels were also observed in an analysis conducted by Najafpour Boushehri $S$, et al. (2020) [19]. In earlier reviews, findings were less conclusive. Aziz Z, et al. (2013) [21] collated evidence from six RCTs involving 474 participants and found no associations between HSL ingestion and total cholesterol, high density lipoprotein cholesterol, low density lipoprotein cholesterol nor triglyceride levels. Hajifaraji M, et al. (2018) [22] observed significant reductions in mean total cholesterol, high density lipoprotein cholesterol and low density lipoprotein cholesterol levels compared to baseline values after participants with genetic (polygenic) dyslipidaemia ingested two cups of sour tea daily. Wahabi HA, et al. (2010) [20] collated data from four trials comprised of 390 patients finding that HSL did not appear to be an effective treatment for primary hypertension, although these trials were regarded to be poor quality.

Regarding human RCTs five focused on blood pressure or hypertension (Table 2) [23-27]. Four reported on lipid levels and profiles $[23,28-30]$. Some RCTs investigated other health outcomes including oxidative stress, [28] insulin resistance, $[23,28]$ and inflammation [23]. Abubakar SM, et al. (2019) [23] researched vascular function and a cardiovascular disease (CVD) risk calculation was undertaken. All the RCTs observed notable improvements in health outcomes, which included improved postprandial vascular function [23], triglyceride levels [29-30], high-density lipoprotein cholesterol levels [28,30], insulin resistance $[23,28]$ and blood pressure profile [24-27].

Of the interventions delivered infusions were generally given one to three times daily. Abubakar SM, et al. (2019) [23] steeped 30g (an average of 15 hibiscus tea bags) of Hibiscus sabdariffa calyces in 1 litre of low nitrate water for $10 \mathrm{~min}$. Each volunteer was then served an equivalent extract of $7.5 \mathrm{~g}$ Hibiscus sabdariffa calyces (about 3-4 hibiscus tea bags) in $250 \mathrm{~mL}$ Buxton water once daily. In four other studies $240 \mathrm{~mL}$ servings were delivered two or three times daily with this appearing to benefit systolic and diastolic blood pressure and triglyceride levels $[25,26,29,30]$. Some studies administered the tea interventions with a date or sugar cube to aid compliance in case the tea flavour was disliked $[26,28]$.

As shown in Table 3 seven studies scored 3 or more after applying the Jadad criteria indicating that they were of moderate to high quality. One study [29] did not mention randomisation methods nor specify whether blinding was undertaken so was graded as lower quality (a score of 2). The three studies that were 'moderate quality' received lower scores because blinding methods were not clearly specified $[24,27,28]$.

Nine laboratory publications were identified; the majority which were murine models (Table 4). Various HSL interventions were used. Five papers reported that they used aqueous extracts, infusions or drinks [31-35]. Earlier work by Hopkins AL, et al. (2013) [36] purported that HSL reduced cholesterol, low-density lipoprotein cholesterol and triglycerides in most diabetic, hyperlipidaemic and normolipidemic laboratory models. Similarly, Lin HH, et al. (2011) [37] evaluated laboratory models using various HSL forms finding that these appeared to benefit atherosclerosis, cancer, diabetes, liver disease and metabolic syndromes.

Two laboratory models $[31,33]$ one fed a high fat diet observed improvements in cardiac function, which was most likely mediated 


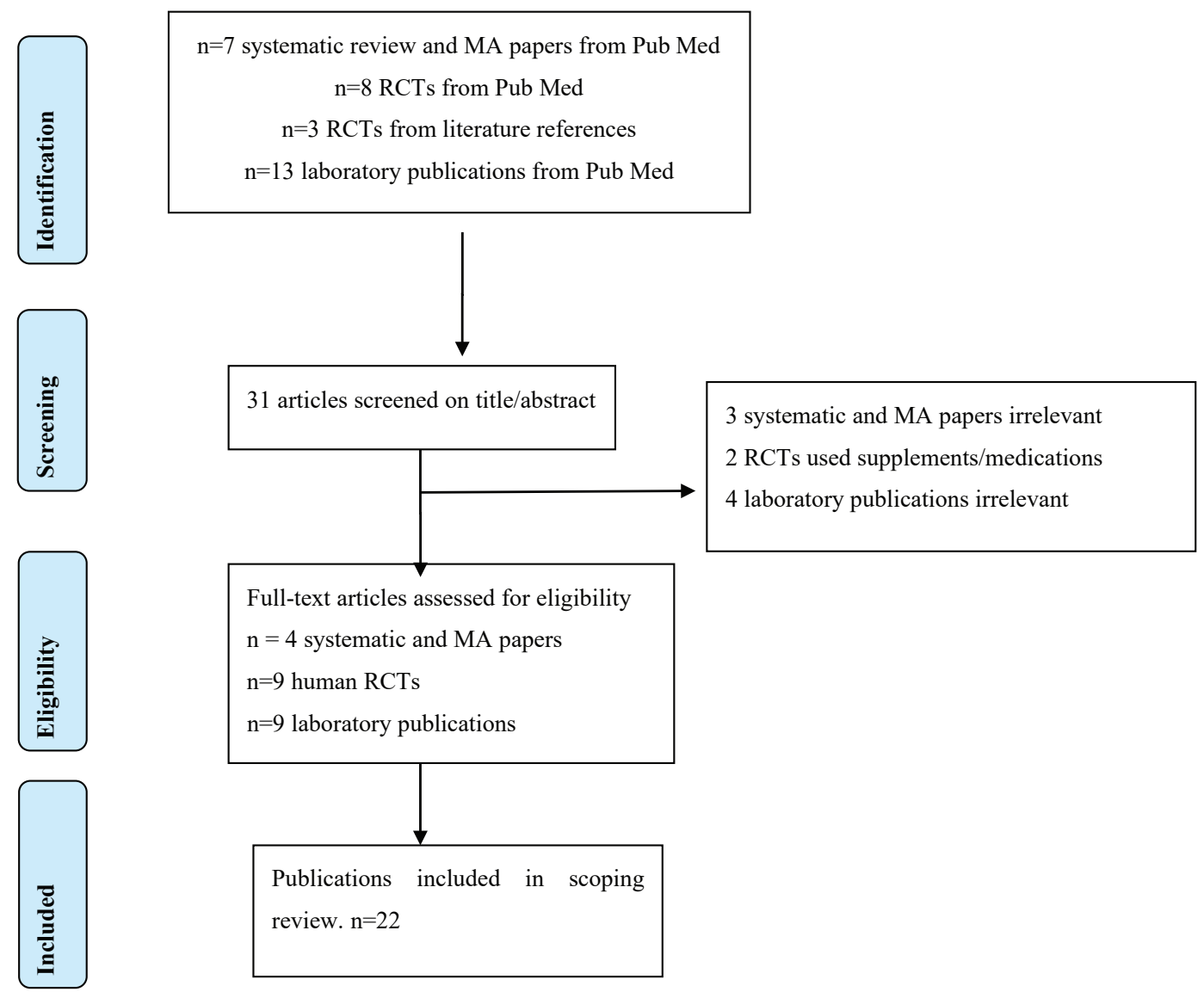

Figure 1: PRISMA approach used to identify studies.

Table 1: Hibiscus tea and health: Evidence from systematic reviews and meta-analysis papers.

\begin{tabular}{|l|c|c|}
\hline \multicolumn{1}{|c|}{ Author } & Health Outcome & Main Finding(s) \\
\hline Najafpour Boushehri S, et al. (2020) [19] & $\begin{array}{c}\text { Glycaemic status, blood } \\
\text { pressure }\end{array}$ & $\begin{array}{c}\text { MA of 7 RCTs showed sour tea consumption significantly reduced fasting } \\
\text { plasma glucose, SBP and DBP. }\end{array}$ \\
\hline Serban C, et al. (2015) [5] & Arterial hypertension & MA of 5 RCTs showed a significant effect of HSL in lowering both SBP and \\
\hline Aziz Z, et al. (2013) [21] & Serum lipids & $\begin{array}{c}\text { Available evidence from 6 RCTs does not support the efficacy of HSL in } \\
\text { lowering serum lipids. }\end{array}$ \\
\hline Wahabi HA, et al. (2010) [20] & Hypertension & $\begin{array}{c}\text { The 4 RCTs identified do not provide reliable evidence to support } \\
\text { recommending HSL for the treatment of primary hypertension in adults. }\end{array}$ \\
\hline
\end{tabular}

*DBP- Diastolic Blood Pressure; HSL- Hibiscus sabdariffa L.; RCTs- Randomised Controlled Trials; SBP- Systolic Blood Pressure

through antioxidant activities. Lee YS, et al. (2018) [38] used a synergistic intervention which included hibiscus flower extracts and found that these reduced obesity in mice fed a high-fat diet. Some work showed that HSL calyces or a roselle drink reduced hepatic and renal inflammation, respectively, possibly by down regulating biochemical components of inflammatory pathways [34,39]. One study [32] reported renal benefits amongst rodents with induced chronic kidney disease effects that were ameliorated in a dose-dependent manner. Surprisingly one study by Mahmoud YI (2012) [35] observed that aqueous extracts from dried HS calyx inversely influenced male murine reproductive fertility but these effects have not since been replicated.
Beyond the span of this scoping review HSL extract has also been found to alter parameters associated with the development of obesity, including cholesterol metabolism, lipid accumulation, adipocyte differentiation and adipogenesis and its inhibitory effects on pancreatic lipase [13]. Some research $[40,41]$ using a combination of HSL polyphenolic extracts and Lippia citrodora Paláu (syn Aloysia citrodora) Verbenaceae observed improved, blood pressure and heart rate parameters amongst obese adults with observed increases in anorexigenic and reductions in orexigenic hormones. Moreover, a multicentric pilot clinical study [42] of 38 adults with blood pressure above $140 / 90 \mathrm{mmHg}$ showed that HSL (10-20 g of HSL per 0.5 litres of tea daily) was helpful in treating uncontrolled hypertension and 
Table 2: Hibiscus tea \& health: Evidence from human trials.

\begin{tabular}{|c|c|c|c|c|c|c|}
\hline $\begin{array}{l}\text { Study (Author, } \\
\text { Year, Location, } \\
\text { Reference } \\
\text { Number) }\end{array}$ & $\begin{array}{l}\text { Subjects (age, } \\
\text { gender) }\end{array}$ & Study design & $\begin{array}{c}\text { Tea Intervention } \\
\text { (type) }\end{array}$ & $\begin{array}{l}\text { Tea Intervention } \\
\text { (dosage) }\end{array}$ & Health Outcome(s) & $\begin{array}{l}\text { Main Findings (with } \\
\text { any reported significant } \\
\text { p-values) }\end{array}$ \\
\hline $\begin{array}{l}\text { Abubakar SM, et al. } \\
\text { (2019) [23] UK }\end{array}$ & $\begin{array}{c}\mathrm{n}=25 \text { with } 1 \% \text { to } 10 \% \\
\text { CVD risk }\end{array}$ & SB RCT & $\begin{array}{l}\text { Aqueous extract } \\
\text { of HSC or water }\end{array}$ & $250 \mathrm{ml}$ & $\begin{array}{l}\text { Postprandial Blood } \\
\text { Pressure, Vascular } \\
\text { Function, Blood } \\
\text { Lipids, Biomarkers } \\
\text { of Insulin } \\
\text { Resistance and } \\
\text { Inflammation }\end{array}$ & $\begin{array}{l}\text { HSC extract improved } \\
\text { postprandial vascular } \\
\text { function and could be a } \\
\text { useful dietary strategy } \\
\text { to reduce endothelial } \\
\text { dysfunction and CVD risk. }\end{array}$ \\
\hline $\begin{array}{l}\text { Hajifaraji M,et al. } \\
\text { (2018) [22] Iran }\end{array}$ & $\begin{array}{c}n=43 \text { adults }(30-60 \\
\text { years old })\end{array}$ & 12-week RCT & Dorsa sour tea & $\begin{array}{c}\text { Two cups of sour } \\
\text { tea daily }\end{array}$ & Dyslipidaemia & $\begin{array}{l}\text { Mean concentration of } \\
\text { total cholesterol, HDL-C } \\
\text { and LDL-C significantly } \\
\text { decreased in the } \\
\text { intervention group in } \\
\text { comparison to their } \\
\text { baseline values. }\end{array}$ \\
\hline $\begin{array}{l}\text { Jeenduang M, } \\
\text { et al. (2017) [29] } \\
\text { Thailand }\end{array}$ & $\begin{array}{l}\text { Subjects with } \\
\text { hypercholesterolemia } \\
n=48\end{array}$ & 30-day trial & $\begin{array}{l}\text { Hibiscus sabdariffa } \\
\text { or Gynostemma } \\
\text { pentaphyllum } \\
\text { Makino tea }\end{array}$ & $\begin{array}{l}\text { The tea sachet ( } 3 \\
\text { g) was added to } \\
240 \mathrm{~mL} \text { of boiling } \\
\text { water and drunk } \\
\text { twice daily. }\end{array}$ & Lipid Levels & $\begin{array}{l}\text { HS consumption may } \\
\text { have beneficial effects } \\
\text { with respect to TG } \\
\text { concentrations in B2 } \\
\text { carriers, but it may } \\
\text { adversely affect HDL-C } \\
\text { concentrations in } \\
\text { homozygous B1B1 and E4 } \\
\text { carriers. }\end{array}$ \\
\hline $\begin{array}{l}\text { Mozaffari-Khosravi } \\
\mathrm{H} \text {, et al. (2014) [28] } \\
\text { Iran }\end{array}$ & $\begin{array}{l}\text { Patients with T2D } \\
\qquad n=100\end{array}$ & 4-week RCT & Sour or green tea & $\begin{array}{l}150 \mathrm{~mL} \text { infusion } \\
\text { three times a day }\end{array}$ & $\begin{array}{l}\text { Lipid Profile, } \\
\text { Oxidative Stress, } \\
\text { Insulin Resistance }\end{array}$ & $\begin{array}{c}\text { Sour tea had positive effect } \\
\text { on insulin resistance and } \\
\text { increased HDL-C. }\end{array}$ \\
\hline $\begin{array}{l}\text { Mozaffari-Khosravi } \\
\text { H, et al. (2013) [24] } \\
\text { Iran }\end{array}$ & $\begin{array}{l}\text { Mildly hypertensive } \\
\text { patients with } \\
\text { diabetes } n=100\end{array}$ & 4-week RCT & Sour or green tea & $\begin{array}{l}\text { Three glasses a } \\
\text { day } 2 \text { hr after } \\
\text { each meal. }\end{array}$ & Blood Pressure & $\begin{array}{c}\text { Drinking three glasses of } \\
\text { sour tea daily for } 4 \text { weeks } \\
\text { significantly decreased SBP } \\
\text { and DBP. }\end{array}$ \\
\hline $\begin{array}{l}\text { McKay DL, et al. } \\
\text { (2010) [25] USA }\end{array}$ & $\begin{array}{c}\text { Pre- and mildly } \\
\text { hypertensive adults, } \\
\mathrm{n}=65\end{array}$ & 6-week DB RCT & $\begin{array}{l}\text { Brewed hibiscus } \\
\text { tea or placebo } \\
\text { beverage }\end{array}$ & $\begin{array}{l}\text { Three } 240 \mathrm{~mL} \\
\text { servings daily } \\
(720 \mathrm{~mL} / \mathrm{d})\end{array}$ & Blood Pressure & $\begin{array}{l}\text { Hibiscus tea lowered SBP } \\
\text { compared with placebo. } \\
\text { DBP was also lower, } \\
\text { although this change did } \\
\text { not differ from placebo. }\end{array}$ \\
\hline $\begin{array}{l}\text { Mozaffari-Khosravi } \\
\text { H, et al. (2009a) } \\
\text { [30] Iran (lipids) }\end{array}$ & $\begin{array}{l}\text { Patients with } \\
\text { diabetes } n=60\end{array}$ & 1-month RCT & Sour or black tea & $\begin{array}{l}240 \mathrm{~mL} \text { twice } \\
\text { daily. }\end{array}$ & $\begin{array}{l}\text { Lipid Profile and } \\
\text { Lipoproteins }\end{array}$ & $\begin{array}{l}\text { In the ST group, HDL-C } \\
\text { increased significantly } \\
\text { and total cholesterol, } \\
\text { LDL-C, TGs and Apo-B100 } \\
\text { decreased. }\end{array}$ \\
\hline $\begin{array}{l}\text { Mozaffari- } \\
\text { Khosravi H, et al. } \\
\text { (2009b) [26] Iran } \\
\text { (hypertension) }\end{array}$ & $\begin{array}{l}\text { Patients with } \\
\text { diabetes and mild } \\
\text { hypertensionn=60 }\end{array}$ & 1-month RCT & Sour or black tea & $\begin{array}{l}240 \mathrm{~mL} \text { twice } \\
\text { daily. }\end{array}$ & Hypertension & $\begin{array}{l}\text { The ST infusion had } \\
\text { positive effects on systolic } \\
\text { BP in type II diabetic } \\
\text { patients with mild } \\
\text { hypertension. }\end{array}$ \\
\hline $\begin{array}{l}\text { Haji Faraji M, et al. } \\
\text { (1999) [27] Iran }\end{array}$ & $\begin{array}{l}\text { Patients with } \\
\text { moderate essential } \\
\text { hypertension } n=54\end{array}$ & 15-day RCT & Sour tea or control & $\begin{array}{l}\text { Instructed to } \\
\text { use one glass of } \\
\text { the decoction } \\
\text { (two spoonfuls } \\
\text { of blended tea } \\
\text { in one glass of } \\
\text { boiled water } \\
\text { boiled for 20-30 } \\
\text { min). }\end{array}$ & Hypertension & $\begin{array}{c}\text { There was an } 11.2 \% \\
\text { lowering of SBP and a } \\
10.7 \% \text { decrease of DBP in } \\
\text { the experimental group } \\
12 \text { days after beginning } \\
\text { treatment. }\end{array}$ \\
\hline
\end{tabular}

*BP- Blood Pressure; CVD- Cardiovascular disease; DB- Double-blind; DBP- Diastolic Blood Pressure; HDL-C- High-Density Lipoprotein Cholesterol; HSLHibiscus sabdariffa Linne; HSC- Hibiscus sabdariffa calyces; LDL-C- Low-Density Lipoprotein Cholesterol; RCT- Randomised Controlled Trial; SB- Singleblind; SBP- Systolic Blood Pressure; ST- sour tea; STE- Sour Tea Extract; TG- Triglyceride. 
Table 3: Jadad criterion used to assess quality of RCTs.

\begin{tabular}{|c|c|c|c|c|c|c|}
\hline Publication & Randomisation & $\begin{array}{c}\text { Method of } \\
\text { Randomisation } \\
\text { Described \& } \\
\text { Appropriate }\end{array}$ & $\begin{array}{l}\text { Blinding } \\
\text { Mentioned }\end{array}$ & $\begin{array}{l}\text { Method of } \\
\text { Blinding Described } \\
\text { and Appropriate }\end{array}$ & $\begin{array}{c}\text { Withdrawal } \\
\text { and Dropout of } \\
\text { Subjects Provided }\end{array}$ & Total Score \\
\hline Abubakar SM, et al. (2019) [23] UK & 1 & 1 & 1 & 0 & 1 & 4 \\
\hline $\begin{array}{l}\text { Jeenduang N, et al. (2017) [29] } \\
\text { Thailand }\end{array}$ & 1 & 0 & 0 & 0 & 1 & 2 \\
\hline $\begin{array}{l}\text { Mozaffari-Khosravi H, et al. (2014) } \\
\text { [28] Iran }\end{array}$ & 1 & 1 & 0 & 0 & 1 & 3 \\
\hline $\begin{array}{l}\text { Mozaffari-Khosravi H, et al. (2013) } \\
\text { [26] Iran }\end{array}$ & 1 & 1 & 0 & 0 & 1 & 3 \\
\hline McKay DL, et al. (2010) [25] USA & 1 & 1 & 1 & 1 & 1 & 5 \\
\hline $\begin{array}{l}\text { Mozaffari-Khosravi H, et al. (2009a) } \\
\text { [30] Iran (lipids) }\end{array}$ & 1 & 1 & 1 & 1 & 1 & 5 \\
\hline $\begin{array}{l}\text { Mozaffari-Khosravi H, et al. (2009b) } \\
\text { [26] Iran (hypertension) }\end{array}$ & 1 & 1 & 1 & 1 & 1 & 5 \\
\hline Haji Faraji M, et al. (1999) [27] Iran & 1 & 1 & 0 & 0 & 1 & 3 \\
\hline
\end{tabular}

Total quality assessment score for which scores range between 1 and 5 : with 1 being the lowest quality and 5 being the highest quality. $3=$ above average quality.

Table 4: Hibiscus Tea and Health: Evidence from Laboratory Publications.

\begin{tabular}{|c|c|c|c|c|}
\hline $\begin{array}{l}\text { Study (Author, Year, } \\
\text { Reference Number) }\end{array}$ & Study Design & $\begin{array}{l}\text { Hibiscus Intervention } \\
\text { (type) }\end{array}$ & Health Outcome(s) & Main Findings \\
\hline $\begin{array}{l}\text { Pérez-Torres I, et al. } \\
\text { (2019) [31] }\end{array}$ & Rat model & HSL infusion & Cardiac function & $\begin{array}{l}\text { Treatment with an infusion from HSL calyces protected } \\
\text { cardiac function from damage by ischemia and } \\
\text { reperfusion through antioxidant activities. }\end{array}$ \\
\hline Lee YS, et al. (2018) [38] & $\begin{array}{l}\text { Obese mouse } \\
\text { model }\end{array}$ & $\begin{array}{l}\text { Combination of lemon } \\
\text { verbena and hibiscus- } \\
\quad \text { flower extracts }\end{array}$ & Obesity & $\begin{array}{l}\text { The formula decreased obesity and its complications in } \\
\text { mice fed a high-fat diet. }\end{array}$ \\
\hline Ali BH, et al. (2017) [32] & Rat model & $\begin{array}{l}\text { Aqueous extract of HSL } \\
\text { calyces ( } 5 \text { and } 10 \%)\end{array}$ & CKD & $\begin{array}{l}\text { Adverse effects of CKD were significantly lessened in a } \\
\text { dose-dependent manner. }\end{array}$ \\
\hline Si LY, et al. (2017) [33] & $\begin{array}{l}\text { Rat model-fed a } \\
\text { high fat diet }\end{array}$ & Roselle aqueous extract & Cardiac function & $\begin{array}{l}\text { Roselle had a protective effect on cardiac dysfunction in } \\
\qquad \mathrm{OB} \text { and } \mathrm{OB}+\mathrm{MI} \text { rats. }\end{array}$ \\
\hline Chou ST, et al. (2016) [34] & Mouse model & Roselle drink & $\begin{array}{l}\text { Inflammation } \\
\text { (renal) }\end{array}$ & $\begin{array}{l}\text { The roselle drink reduced LPS-induced renal } \\
\text { inflammation by down regulating the cytokine network, } \\
\text { pro-inflammatory product production, and NF-kB } \\
\text { pathways. }\end{array}$ \\
\hline $\begin{array}{l}\text { Ezzat SM, et al. (2016) } \\
\text { [39] }\end{array}$ & $\begin{array}{l}\text { Rat model } \\
\text { with induced } \\
\text { hepatotoxicity }\end{array}$ & HSL calyces & $\begin{array}{l}\text { Inflammation } \\
\text { (hepatic) }\end{array}$ & $\begin{array}{l}\text { The intervention decreased hepatic inflammatory } \\
\text { markers, including tumour necrosis factor alpha, } \\
\text { interleukin-6 and interferon gamma by } 85.4,14.9 \text { and } \\
70.9 \% \text {, respectively. }\end{array}$ \\
\hline $\begin{array}{l}\text { Hopkins AL, et al. (2013) } \\
\text { [36] }\end{array}$ & $\begin{array}{l}\text { Review of } \\
\text { laboratory } \\
\text { studies }\end{array}$ & HSL & Lipid profile & $\begin{array}{l}\text { Total cholesterol, LDL-C and TGs } \\
\text { Reduced in most normolipidaemic, hyperlipidaemic, } \\
\text { and diabetic laboratory models, whereas HDL-C was not } \\
\text { affected by the consumption of HSL extract. }\end{array}$ \\
\hline $\begin{array}{l}\text { Mahmoud YI, et al. } \\
\text { (2012) [35] }\end{array}$ & $\begin{array}{l}\text { Mouse model - } \\
\text { male albino mice }\end{array}$ & $\begin{array}{l}\text { Cold or boiled Hibiscus } \\
\text { aqueous extract or } \\
\text { water. }\end{array}$ & $\begin{array}{l}\text { Reproductive } \\
\text { function }\end{array}$ & $\begin{array}{l}\text { Aqueous extracts from dried calyx of HS, either cold } \\
\text { or boiled appeared to adversely influence male } \\
\text { reproductive fertility in albino mice. }\end{array}$ \\
\hline Lin HH, et al. (2011) [37] & $\begin{array}{l}\text { Evaluation of } \\
\text { laboratory } \\
\text { models (rodents } \\
\text { and rabbits) }\end{array}$ & Various HSL extracts. & $\begin{array}{c}\text { Atherosclerosis, } \\
\text { liver disease, cancer, } \\
\text { diabetes, metabolic } \\
\text { syndromes. }\end{array}$ & $\begin{array}{l}\text { Various HSL extracts exhibit activities against } \\
\text { atherosclerosis, liver disease, cancer, diabetes and other } \\
\text { metabolic syndromes. }\end{array}$ \\
\hline
\end{tabular}

*CKD- Chronic Kidney Disease; HDL-C- High-Density Lipoprotein Cholesterol; HPAIVs- Highly Pathogenic Avian Influenza Viruses; HSL- Hibiscus sabdariffa L.; LPAIVs- Low Pathogenic AIVs; LDL-C- Low-Density Lipoprotein Cholesterol; LPS- Lipopolysaccharide; MI- Myocardial infarction; NF-kB- Nuclear FactorKB; OB- Obese; TGs- Triglyceride. 
well tolerated. Work conducted with soccer players also demonstrated that $450 \mathrm{mg} / \mathrm{d}$ sour tea extracts after 6-weeks had beneficial effects on oxidative stress [43].

\section{Discussion}

The scoping review of scientific evidence found the strongest evidence-base for HSL and cardiovascular wellbeing. Three synthetic reviews, which included MA research, reported benefits for blood pressure and hypertension $[5,19,20]$. Five underpinning human RCTs also consistently observed improvements in blood pressure, particularly systolic blood pressure [23,25-28]. Evidence for serum lipids was less certain. For example, Hajifaraji M, et al. (2018) [22] concluded that sour tea could impact beneficially and significantly on subjects with polygenic dyslipidaemia, which could partly be attributed to its anthocyanin content and the genetic status of subjects. In contrast, earlier work undertaken by Aziz Z, et al. (2013) [21] found that evidence from the six RCTs analysed could not identify definite links between HSL and reduced serum lipids. Authors, however, reported that studies had small sample sizes and were underpowered which could have been attributed to the lack of findings.

It should also be considered that most studies recruited participants with CVD risk factors, hypercholesterolemia, hypertension or type 2 diabetes at baseline, thus were not healthy. This indicates that hibiscus tea consumption could be a useful adjunctive therapy from a protective health stance, particularly amongst individuals at risk of cardiovascular disease. Most human studies provided two-three servings of sour or brewed hibiscus tea daily with a serving typically being $240-250 \mathrm{~mL}$ [24-26,28-30]. Thus it would be reasonable to suggest that hibiscus tea drinking could be adopted as a dietary strategy to enhance individuals with elevated blood pressure or CVD risk. It has previously been reported that HSL extracts have a low degree toxicity and excellent safety tolerability $[10,36]$ although some caution may be required if planning to conceive or pregnant $[35,44]$.

In terms of potential mechanisms, it is well documented that the polyphenol profile of hibiscus tea could play a role in its health benefits and potential modern therapeutic uses [1]. Regarding cardiovascular benefits it has been postulated that the antioxidant effects of HSL induced by its anthocyanin profile could inhibit low-density lipoprotein oxidation, subsequently impeding atherosclerosis [36]. Other theories include that anthocyanins reduce low-density lipoprotein oxidation and inhibit adipogenesis via the regulation of transcription factors and adipogenic signalling pathways, which modulate the gene expression of certain microRNAs [45]. Recent work with type 1 diabetic rats has further shown that HSL renews pancreatic $\beta$-cells [46]. Laboratory publications suggest potential cardiac $[31,35]$ and anti-inflammatory actions $[34,39]$. There was emerging evidence from human trials that hibiscus tea could have beneficial effects on insulin resistance $[23,28]$ and levels of oxidative stress [28]. Ongoing human RCTs are now needed to focus on these aspects as primary research outcomes.

Other work has focused on how HSL could affect parameters of nutritional status. Research conducted on patients with vitamin $B_{12}$ deficiency and neurological symptoms who were given a combination of hydroxocobalamin and HSL found that vitamins injections were no longer required to replete $B_{12}$ status [47]. Extended research by this team of scientists using laboratory models confirmed that the bioavailability of intestinal hydroxocobalamin increased when synergistically used with HSL [47]. Ongoing work is now needed to replicate findings and further determine whether hibiscus tea could improve the bioavailability of nutrients, such as vitamin $\mathrm{B}_{12}$-findings that could potentially be of benefit to vegetarian or vegan populations.
Elsewhere it has been observed that the iron and organic acid profile of HSL could potentially improve hematopoietic (iron status) parameters but larger human studies are needed $[48,49]$.

With respect to limitations it should be considered that studies varied in terms of duration, forms of interventions and comparators used. Future studies should continue to use interventions that ultimately translate into public health guidance. Undoubtedly, more welldesigned RCTs are needed using 'phytochemically characterised' preparations, which would aid comparative analyses between studies $[10,50]$. It must be indicated that future trials should better include and describe blinding methods to improve their quality. Clear randomisation approaches should also be adopted and outlined in publications. Certain populations such as pregnant mothers and those with kidney problems may be cautious to avoid excessive consumption of hibiscus infusions due to these being a source of aluminium which could accumulate in the body [51]. More research is also needed to better understand the toxicology of hibiscus as the ingestion of condensed HSL extracts would be somewhat different to that of infusions-excessive doses of HSL calyx doses, for example, over long durations have been found to have deleterious effects on testes of rats [3].

\section{Conclusions}

The present scoping review shows that hibiscus tea ingestion could benefit cardiovascular wellbeing. Emerging research also reveals potential benefits in terms of its ability to favourably modulate lipid profiles, inflammation, oxidative stress and insulin resistance. From a habitual perspective hibiscus tea could be consumed for its favourable phytonutrient profile and potential cardiovascular benefits. More extensive research is now warranted in the form of larger and longer human trials with clearly defined interventions.

\section{Disclosure}

The views expressed are those of the authors alone and personnel from the UK TEA \& INFUSIONS ASSOCIATION (UKTIA) had no role in writing this review.

\section{Conflicts of Interest}

The authors declare no conflicts of interest.

\section{Acknowledgements}

The authors received funding provided by the Tea Advisory Panel which is supported by an unrestricted educational grant from the UK TEA \& INFUSIONS ASSOCIATION (UKTIA), the trade association for the UK tea industry. UKTIA plays no role in producing the outputs of the panel. Independent panel members include nutritionists, biochemists, dietitians, dentist and doctors. See www. teaadvisorypanel.com

\section{References}

1. Riaz G, Chopra R (2018) A Review on Phytochemistry and Therapeutic Uses of Hibiscus Sabdariffa L. Biomed Pharmacother 102: 575-586.

2. El-Saidy SM, Ismail IA, EL-Zoghbi M (1992) A study on Roselle extraction as a beverage or as a source for anthocyanins. Zagazig J Agric Res 19: 831-839.

3. Ali BH, Al Wabel N, Blunden G (2005) Phytochemical, Pharmacological and Toxicological Aspects of Hibiscus Sabdariffa L.: A Review. Phytother Res 19: 369-375.

4. Wu HY, Yang KM, Chiang PY (2018) Roselle Anthocyanins: Antioxidant Properties and Stability to Heat and pH. Molecules 23: 1357. 
5. Serban C, Sahebkar A, Ursoniu S, Andrica F, Banach M (2015) Effect of Sour Tea (Hibiscus sabdariffa L.) on Arterial Hypertension: A Systematic Review and Meta-Analysis of Randomized Controlled Trials. J Hypertens 33: 1119-1127.

6. Hassan ST, Berchova K, Šudomová M (2016) Antimicrobial, Antiparasitic and Anticancer Properties of Hibiscus sabdariffa (L.) and Its Phytochemicals: In Vitro and in Vivo Studies. Ceska Slov Farm 65: 10-14.

7. Jabeur I, Pereira E, Barros L, Calhelha RC, Soković M, et al. (2017) Hibiscus sabdariffa L. As a Source of Nutrients, Bioactive Compounds and Colouring Agents. Food Res Int 100: 717-723.

8. Wright $\mathrm{Cl}$, Van-Buren L, Kroner $\mathrm{Cl}$, Koning MMG (2007) Herbal Medicines as Diuretics: A Review of the Scientific Evidence. J Ethnopharmacol 114: 1-31.

9. Poswal FS, Russell G, Mackonochie M, MacLennan E, Adukwu EC, et al. (2019) Herbal Teas and their Health Benefits: A Scoping Review. Plant Foods Hum Nutr 74: 266-276.

10. Da-Costa-Rocha I, Bonnlaender B, Sievers H, Pischel I, Heinrich M (2014) Hibiscus sabdariffa L.-A Phytochemical and Pharmacological Review. Food Chem 165: 424-443.

11. Piovesana A, Rodrigues E, Norena CPZ (2019) Composition Analysis of Carotenoids and Phenolic Compounds and Antioxidant Activity From Hibiscus Calyces (Hibiscus sabdariffa L.) by HPLC-DAD-MS/MS. Phytochem Anal 30: 208-217.

12. Gamboa-Gómez $\mathrm{Cl}$, Rocha-Guzmán NE, Gallegos-Infante JA, Moreno-Jiménez MR, Vázquez-Cabral BD, et al. (2015) Plants With Potential Use on Obesity and Its Complications. EXCLI J 14: 809-831.

13. Ojulari OV, Lee SG, Nam JO (2019) Beneficial Effects of Natural Bioactive Compounds from Hibiscus sabdariffa L. on Obesity. Molecules 24: 210.

14. Walton RJ, Whitten DL, Hawrelak JA (2016) The efficacy of Hibiscus sabdariffa (rosella) in essential hypertension: a systematic review of clinical trials. Aus J Herbal Med 28: 48-51.

15. Tricco AC, Lillie E, Zarin W, O'Brien KK, Colquhoun H, et al. (2018) PRISMA Extension for Scoping Reviews (PRISMA-ScR): Checklist and Explanation. Ann Intern Med 169: 467-473.

16. Moher D, Shamseer L, Clarke M, Ghersi D, Liberati A, et al. (2015) Preferred Reporting Items for Systematic Review and Meta-Analysis Protocols (PRISMA-P) 2015 Statement. Syst Rev 4: 1.

17. Jadad AR, Moore RA, Carroll D, Jenkinson C, Reynolds DJ, et al. (1996) Assessing the Quality of Reports of Randomized Clinical Trials: Is Blinding Necessary? Control Clin Trials 17: 1-12.

18. Hariton E, Locascio JJ (2018) Randomised Controlled Trials - The Gold Standard for Effectiveness Research: Study Design: Randomised Controlled Trials. BJOG 125:1716.

19. Boushehri NS, Karimbeiki R, Ghasempour S, Ghalishourani SS, Pourmasoumi M, et al. (2020) The Efficacy of Sour Tea (Hibiscus sabdariffa L.) on Selected Cardiovascular Disease Risk Factors: A Systematic Review and Meta-Analysis of Randomized Clinical Trials. Phytother Res 34: 329-339.

20. Wahabi HA, Alansary LA, Al-Sabban AH, GlasziuoP (2010) The Effectiveness of Hibiscus Sabdariffa in the Treatment of Hypertension: A Systematic Review. Phytomedicine 17: 83-86.

21. Aziz Z, Wong SY, Chong NJ (2013) Effects of Hibiscus sabdariffa L. On Serum Lipids: A Systematic Review and Meta-Analysis. J Ethnopharmacol 150: 442-450.
22. Hajifaraji M, Matlabi M, Ahmadzadeh-Sani F, Mehrabi Y, Rezaee MS, et al. (2018) Effects of Aqueous Extracts of Dried Calyx of Sour Tea (Hibiscus sabdariffa L.) on Polygenic Dyslipidemia: A Randomized Clinical Trial. Avicenna J Phytomed 8: 24-32.

23. Abubakar SM, Ukeyima MT, Spencer JPE, Lovegrove JA (2019) Acute Effects of Hibiscus Sabdariffa Calyces on Postprandial Blood Pressure, Vascular Function, Blood Lipids, Biomarkers of Insulin Resistance and Inflammation in Humans. Nutrients 11: 341.

24. Mozaffari-Khosravi H, Ahadi Z, Barzegar K (2013) The Effect of Green Tea and Sour Tea on Blood Pressure of Patients With Type 2 Diabetes: A Randomized Clinical Trial. J Diet Suppl 10: 105-115.

25. McKay DL, Chen CY, Saltzman E, Blumberg JB (2010) Hibiscus Sabdariffa L. Tea (Tisane) Lowers Blood Pressure in Prehypertensive and Mildly Hypertensive Adults. J Nutr 140: 298-303.

26. Mozaffari-Khosravi H, Jalali-Khanabadi BA, Afkhami-Ardekani M, Fatehi F, Noori-Shadkam M (2009) The Effects of Sour Tea (Hibiscus sabdariffa) on Hypertension in Patients With Type II Diabetes. J Hum Hypertens 23: 48-54.

27. Faraji MH, Tarkhani AH (1999) The Effect of Sour Tea (Hibiscus sabdariffa) on Essential Hypertension. J Ethnopharmacol 65: 231 236.

28. Mozaffari-Khosravi H, Ahadi Z, Tafti MF (2014) The Effect of Green Tea versus Sour Tea on Insulin Resistance, Lipids Profiles and Oxidative Stress in Patients with Type 2 Diabetes Mellitus: A Randomized Clinical Trial. Iran J Med Sci 39: 424-432.

29. Jeenduang N, Sangkaew B, Chantaracha P, Chanchareonsri S, Plyduang T, et al. (2017) APOE and CETP TaqIB Polymorphisms Influence Metabolic Responses to Hibiscus sabdariffa L. And Gynostemma Pentaphyllum Makino Tea Consumption in Hypercholesterolemic Subjects. Asia Pac J Clin Nutr 26: 368-378.

30. Mozaffari-Khosravi H, Jalali-Khanabadi BA, Afkhami-Ardekani $M$, Fatehi $F$ (2009) Effects of Sour Tea (Hibiscus sabdariffa) on Lipid Profile and Lipoproteins in Patients With Type II Diabetes. J Altern Complement Med 15: 899-903.

31. Perez-Torres I, Torres-Narvaez JC, Guarner-Lans V, Díaz-Díaz E, Perezpeña-Diazconti M, et al. (2019) Myocardial Protection from Ischemia-Reperfusion Damage by the Antioxidant Effect of Hibiscus sabdariffa Linnaeus on Metabolic Syndrome Rats. Oxid Med Cell Longev 2019: 1724194.

32. Ali BH, Cahlikova L, Opletal L, Karaca T, Manoj P, et al. (2017) Effect of Aqueous Extract and Anthocyanins of Calyces of Hibiscus sabdariffa (Malvaceae) in Rats With Adenine-Induced Chronic Kidney Disease. J Pharm Pharmacol 69: 1219-1229.

33. Si LYN, Ali SAM, Latip J, Fauzi NM, Budin SB, et al. (2017) Roselle Is Cardioprotective in Diet-Induced Obesity Rat Model With Myocardial Infarction. Life Sci 191: 157-165.

34. Chou ST, Lo HY, Li CC, Cheng LC, Chou PC, et al. (2016) Exploring the Effect and Mechanism of Hibiscus sabdariffa on Urinary Tract Infection and Experimental Renal Inflammation. J Ethnopharmacol 194: 617-625.

35. Mahmoud YI (2012) Effect of Extract of Hibiscus on the Ultrastructure of the Testis in Adult Mice. Acta Histochem 114: 342-348.

36. Hopkins AL, Lamm MG, Funk JL, Ritenbaugh C (2013) Hibiscus sabdariffa L. In the Treatment of Hypertension and Hyperlipidemia: A Comprehensive Review of Animal and Human Studies. Fitoterapia 85: 84-94. 
37. Lin $\mathrm{HH}$, Chen JH, Wang CJ (2011) Chemopreventive properties and molecular mechanisms of the bioactive compounds in Hibiscus sabdariffa Linne. Curr Med Chem 18: 1245-1254.

38. Lee YS, Yang WK, Kim HY, Min B, Caturla N, et al. (2018) Metabolaid Combination of Lemon Verbena and Hibiscus Flower Extract Prevents High-Fat Diet-Induced Obesity through AMP-Activated Protein Kinase Activation. Nutrients 10: 1204.

39. Ezzat SM, Salama MM, El-Din SHS, Saleh S, El-Lakkany NM, et al. (2016) Metabolic Profile and Hepatoprotective Activity of the Anthocyanin-Rich Extract of Hibiscus sabdariffa calyces. Pharm Biol 54: 3172-3181.

40. Boix-Castejon M, Herranz-Lopez M, Gago AP, Olivares-Vicente M Caturla N , et al. (2018) Hibiscus and Lemon Verbena Polyphenols Modulate Appetite-Related Biomarkers in Overweight Subjects: A Randomized Controlled Trial. Food Funct 9: 3173-3184.

41. Boix-Castejon M, Herranz-Lopez M, Gago AP, Olivares-Vicente M, Caturla N, et al. (2018) Correction: Hibiscus and Lemon Verbena Polyphenols Modulate Appetite-Related Biomarkers in Overweight Subjects: A Randomized Controlled Trial. Food Funct 9: 4037.

42. Al-Anbaki M, Nogueira RC, Cavin AL, Al-Hadid M, Al-Ajlouni I, et al. (2019) Treating Uncontrolled Hypertension with Hibiscus sabdariffa When Standard Treatment Is Insufficient: Pilot Intervention. J Altern Complement Med 25: 1200-1205.

43. Hadi A, Pourmasoumi M, Kafeshani M, Karimian J, Maracy MR, et al. (2017) The Effect of Green Tea and Sour Tea (Hibiscus Sabdariffa L.) Supplementation on Oxidative Stress and Muscle Damage in Athletes. J Diet Suppl 14: 346-357.
44. de Arruda A, Cardoso CAL, Vieira M do C, Arena CA (2016) Safety assessment of Hibiscus sabdariffa after maternal exposure on male reproductive parameters in rats. Drug Chem Toxicol 39: 22-27.

45. Guardiola S, Mach N (2014) Therapeutic Potential of Hibiscus sabdariffa: A Review of the Scientific Evidence. Endocrinol Nutr 61: 274-295.

46. Adeyemi DO, Adewole OS (2019) Hibiscus sabdariffa Renews Pancreatic $\beta$-cells in Experimental Type 1 Diabetic Model Rats. Morphologie 103: 80-93.

47. Souirti Z, Loukili M, Soudy ID, Rtibi K, Özel A, et al. (2016) Hibiscus Sabdariffa Increases Hydroxocobalamin Oral Bioavailability and Clinical Efficacy in Vitamin $B_{12}$ Deficiency With Neurological Symptoms. Fundam Clin Pharmacol 30: 568-576.

48. Peter EL, Rumisha SF, Mashoto KO, Minzi OM, Mfinanga S (2017) Efficacy of Standardized Extract of Hibiscus sabdariffa L. (Malvaceae) in Improving Iron Status of Adults in Malaria Endemic Area: A Randomized Controlled Trial. J Ethnopharmacol 209: 288-293.

49. Kubuga CK, Hong HG, Song WO (2019) Hibiscus sabdariffa Meal Improves Iron Status of Childbearing Age Women and Prevents Stunting in Their Toddlers in Northern Ghana. Nutrients 11: 198.

50. Carvajal-Zarrabal O, Barradas-Dermitz DM, Orta-Flores Z, HaywardJones PM, Nolasco-Hipólito C, et al. (2012) Hibiscus Sabdariffa L., Roselle Calyx, From Ethnobotany to Pharmacology. J Exp Pharmacol 4: 25-39.

51. Frankova A, Malik J, Drabek O, Szakova J, Sperlingova I, et al. (2016) In Vitro Digestibility of Aluminum from Hibiscus sabdariffa Hot Watery Infusion and Its Concentration in Urine of Healthy Individuals. Biol Trace Elem Res 174: 267-273. 